\title{
The Influence of Patient Experience with Healthcare on the Health-Related Quality of Life of People Living with HIV: An Observational Cross-Sectional Survey
}

\author{
María J. Fuster-RuizdeApodaca (D) · Nuria Sánchez-Vega • \\ María J. Galindo · Ignacio Marín-Jimenez · Javier de Toro • \\ Domingo Orozco-Beltrán · Manuel Cotarelo · Juan Carlos López
}

Received: May 30, 2019 / Published online: July 9, 2019

(C) The Author(s) 2019

\begin{abstract}
Introduction: Patient experience is central to the quality of healthcare delivery, showing positive associations with several outcome measures. The main objectives of this study are to analyze the influence of patient experience on the health-related quality of life in people living with HIV and the role played by treatment complexity and clinical care.
\end{abstract}

Enhanced digital features To view enhanced digital features for this article go to https://doi.org/10.6084/ m9.figshare.8342825.

Electronic supplementary material The online version of this article (https://doi.org/10.1007/s40121019-0252-3) contains supplementary material, which is available to authorized users.

M. J. Fuster-RuizdeApodaca ( $₫) \cdot$ M. J. Galindo . J. C. López

Research Department, SEISIDA (Spanish AIDS

Multidisciplinary Society), Madrid, Spain

e-mail: gerencia@seisida.net

N. Sánchez-Vega · M. Cotarelo

Medical Affairs Department, Merck Sharp and

Dohme Spain, Madrid, Spain

M. J. Galindo

Internal Medicine Department, Clinic University

Hospital, Valencia, Spain

I. Marín-Jimenez

IBD Unit, Gastroenterology Department Clinical Research Institute Gregorio Marañón (IiSGM),

Gregorio Marañón Hospital, Madrid, Spain
Methods: We conducted a cross-sectional survey with 467 patients with HIV. We used the Instrument for Evaluation of the Experience of Chronic Patients and the Health-related Quality of Life Questionnaire (EQ-5D-5L). We analyzed a predictive model through the partial least squares (PLS) method.

Results: The patient self-management scores showed the highest positive relationship with the patient's health-related quality of life $(\beta=0.24, \beta=0.32, p<0.0001)$. Patients' treatment complexity had a negative influence on health-related quality of life $(\beta=-0.21$, $\beta=-0.28, \quad p<0.0001)$. The complexity of clinical care had negative effects on health-related quality of life, both directly $(\beta=-0.37$, $\beta=-0.19, p<0.0001)$ and through its negative influence on the productive interactions

J. de Toro

Rheumatology Department, A Coruña University Hospital, A Coruña, Spain

D. Orozco-Beltrán

Clinical Medicine Department, Miguel Hernandez University, San Juan de Alicante, Spain

J. C. López

HIV Unit, Clinical Microbiology and Infectious Diseases Department, Clinical Research Institute Gregorio Marañón (IiSGM), Gregorio Marañón Hospital, Madrid, Spain 
with healthcare professionals $(\beta=-0.21$, $p<0.0001)$ and patient self-management factors $(\beta=-0.21, p<0.0001)$. The effects of patient experience dimensions on their healthrelated quality of life were higher in people living with HIV $>50$ years old $(p<0.05)$.

Conclusions: Patient experience mainly influenced the health-related quality of life of older people living with HIV. The treatment and clinical care complexity played an important role in degrading the patients' experience and their quality of life. More integrated care would benefit the health-related quality of life of people living with HIV.

Funding: This project was funded by Merck Sharp \& Dohme, Spain.

Keywords: Health-related quality of life; HIV; Patient experience

\section{INTRODUCTION}

An estimated 36.7 million people are living with HIV worldwide [1], of which approximately 145,000 live in Spain [2]. Improvements in antiretroviral therapy (ART) have led to increased life expectancy for many people living with HIV. In the last 2 decades, HIV infection has changed from a subacute infection with a high mortality rate to a chronic manageable infection. As a consequence, there has been a gradual increase in the number of aging people living with HIV [3]. In 2016, 46.3\% of people living with HIV in Spain were $\geq 50$ years of age $[4,5]$. In this scenario, AIDS-defining illnesses have lost their leading role and have been displaced by the different comorbidities related to age and the geriatric syndromes themselves [4]. However, several HIV-related problems still have a notable impact on health-related quality of life, even in virally suppressed people living with HIV as a result of taking ART [6], and this could have a negative impact in their health outcomes [7].

To address the wellbeing of people living with HIV constitutes a big challenge for healthcare systems [8]. It implies improving the model of care to face the complexity of addressing not only the combined burden of HIV and its comorbidities [4, 9] but also other issues related to the health-related quality of life of people living with HIV [8]. This requires a patient-centered approach that involves patient engagement as well as the widespread use of innovations in chronic care [10]. In this context, having information about the patient's experience can provide relevant information to improve the quality of care [10-12]. Patient experience is defined as the information that patients provide to facilitate their continued interaction with health and social care professionals and services and their experience of that interaction and its outcomes [10]. Patient experience has been recognized as a central issue in the quality of healthcare delivery to patients along with clinical effectiveness and patient safety across a wide range of disease areas [12].

The present study is a secondary analysis of broader research that has evaluated the experience of chronically ill patients (diabetes mellitus, HIV, inflammatory bowel disease, and rheumatic diseases) with healthcare. For this purpose, it used the Instrument for Evaluation of the Experience of Chronic Patients (IEXPAC) Questionnaire [10]. This scale is one of the most recent instruments developed, which incorporates a broad notion of integrated care. Results of the principal analysis showed that people living with HIV presented better overall experience with healthcare than patients with other chronic conditions. It also revealed that treatment and clinical care complexity had a negative association with better patient experience. The primary analysis was described elsewhere [13].

The present analysis aimed to go one step further, analyzing the influence of the different dimensions of patient experience on the healthrelated quality of life of people living with HIV. To our knowledge, hardly any studies have assessed such influence. We also analyzed the role played by the treatment and clinical care complexity of people living with HIV in the relationship between patient experience and health-related quality of life. 


\section{METHODS}

\section{Study Population and Procedures}

We conducted a cross-sectional survey in which 467 people living with HIV participated. They were recruited by convenience sampling. The general inclusion criteria were: HIV+ diagnosis, $\geq 18$ years, and having multiple morbidities. The present study is part of broader research that evaluated chronically ill patients' experience with healthcare [13].

HIV care in Spain is provided in tertiary care settings (i.e., public hospitals) through specific HIV clinics within the infectious diseases units. Antiretroviral drugs are dispensed in hospital pharmacies by specialized personnel. Access to the Spanish healthcare system is universal, and almost all patients are treated in hospitals that belong to the public healthcare system. People living with HIV may be attended in a primary care setting for conditions attended in primary care (i.e., non-HIV-related comorbidities). HIV specialists from 25 HIV clinics across Spain collaborated in the recruitment of participants. During their consultations, they explained the goal of the study to the patients and proposed their participation. They requested them to complete the survey voluntarily and anonymously at home and then return it by pre-paid mail.

To minimize selection bias, each physician offered participation in the study to the first 25 consecutive patients attending the clinic routinely, regardless of age, sex, disease severity, or any other criteria. Survey distribution and collection were conducted between May and September 2017. The survey was handed to 627 patients and was returned by 467 patients (response rate of $74.72 \%$ ).

The Clinical Investigation Ethics Committee of the Gregorio Marañon Hospital of Madrid (Spain) approved the research protocol. The study documentation included printed instructions and information for patients about the anonymous nature of the survey and aggregated data processing, which ensured that patient identification was not possible. As agreed by the Clinical Investigation Ethics Committee, the voluntary return of completed questionnaires was taken as implied consent to participate in the study. No clinical data were collected in this study.

\section{Instrument}

The survey contained the following variables: Instrument for Evaluation of the Experience of Chronic Patients (IEXPAC) [10]. It includes 11 items, plus an additional item for recently hospitalized patients. The items refer to the last 6 months, except for the question about hospitalization, which refers to the previous 3 years, and they have a five-option Likert scale response (always, mostly, sometimes, seldom, and never). The first 11 items have a three-factor structure. Factor 1 is called productive interactions and refers to the characteristics and content of interactions between patients and professionals designed to improve outcomes. Factor 2, the new relational model, refers to new forms of patient interactions with the healthcare system, through the internet, or with peers. Finally, factor 3, called patient self-management, refers to individuals' ability to cope with their diseases, manage their self-care, and improve their wellbeing, based on interventions mediated by healthcare professionals. The results of the Spanish validation study of this instrument showed good fit indices (SMRS = 0.05 , GFI $=0.96$, and $\mathrm{CFI}=0.96$ ) and reliability of the factors $(\mathrm{PI}=0.91, \mathrm{NRM}=0.90$, and $\mathrm{PSM}=$ 0.95) [10].

Health-related Quality of Life Questionnaire (EQ-5D-5L) [14, 15]: The EQ-5D-5L is a standardized generic measure that comprises five dimensions (mobility, self-care, usual activities, pain/discomfort, and anxiety/depression) with five levels each (no problems, slight problems, moderate problems, severe problems, and extreme problems). The instrument also contains a visual analog scale (EQ-VAS) that records the respondent's self-rated health on a $20-\mathrm{cm}$ vertical scale with end points labeled "the best you can imagine" and "the worst you can imagine." We used the validated Spanish version of this measure.

The survey also contains the following variables: (1) complexity of clinical care-number of different physicians seeing the patient and number of different healthcare sites that the 
patient usually attends; (2) complexity of the patient's treatment-number of different medicines that the patient takes and the number of daily doses of the treatment the patient takes. Finally, the survey collected primary general demographic data (sex, age, educational level, and current occupational status).

The survey can be consulted in the Electronic Supplementary Material of Orozco-Beltrán et al. [13]. All the survey was designed and administered in Spanish.

\section{Data Analysis}

First, we performed an exploratory analysis. Next, we analyzed differences in the target variables according to participants' gender and age. Regarding age, we established the cutoff point, generally accepted in HIV, of 50 years to consider the health impact of aging in people living with HIV [4] (group $1 \leq 49$ years, group $2 \geq 50$ years).

Following that, we adopted a predictive model based on correlations between the studied variables and previous results of our research [13] through the partial least squares (PLS) method, using the SmartPLS 3.0 software. The partial least squares is a variance-based technique recommended at an early stage of the theoretical development to test and validate exploratory models $[16,17]$. In the partial least squares analysis, the relationships among the studied constructs were analyzed using linear regression in which the loads could be interpreted as standardized beta coefficients. The confidence intervals were based on bootstrapping 500 samples that allowed the generalization of the results and the computation of Student's $t$ for each hypothesis [18]. The coefficient of explained variance $\left(R^{2}\right)$ of each endogenous latent variable should be $>0.10$ [19]. No assumption of normality of the distributions is required [20].

To assess the specific contribution of the variables studied, we first determined whether both measures of health-related quality of life (EQ-5D-5L and EQ-VAS) could be predicted by the factors of the Instrument for Evaluation of the Experience of Chronic Patients. Second, we introduced treatment complexity in the model.
Next, we introduced the complexity of clinical care. We assessed the effect sizes of the models tested through the $f^{2}$ statistic. Values of 0.02 , 0.15 , and 0.35 indicate small, medium, and large effects, respectively [17].

Finally, we evaluated the effect of aging in the model, comparing the final model in the two agespecific groups established ( $\mathrm{G} 1 \leq 49$ years, $\mathrm{G} 2 \geq 50$ years). For this purpose, we first conducted the measurement invariance of composite models (MICOM) procedure in PLS. If the results support the measurement invariance, a multigroup analysis could be conducted to compare the path coefficients of the structural model. However, if the measurement invariance is not established, a multigroup analysis is not feasible, and thus it is recommended to analyze the groups separately [21].

\section{RESULTS}

Most participants were male (73.5\%). Mean age of the participants was $51.6(\mathrm{SD}=10.9)$. A total of $44.3 \%$ were working, $34.3 \%$ were retired or occupationally disabled, $16.7 \%$ were unemployed, and $4.8 \%$ had another occupation (student or housework). The majority of the participants had studied primary $(42.4 \%)$ or secondary education (35.9\%), and $21.7 \%$ had a university degree.

\section{Correlations Among the Variables Under Study and Differences According to Participants' Characteristics}

The descriptive statistics of the variables used in the model, as well as the correlations among the variables, are shown in Table 1.

Results showed that female patients' selfmanagement score was higher than that of males $(4.24 \pm 0.66$ vs. $4.08 \pm 0.82, t=-1.97$, $p=0.50$, Cohen's $d=0.20)$. Regarding age, those older than $50(n=227)$ presented significantly higher scores in the productive interactions factor of the Instrument for Evaluation of the Experience of Chronic Patients than those younger than 49 years $(n=146),(4.54 \pm 0.067$, $t=2.09$ vs. $4.37 \pm 0.075 ; t=2.09, p=0.37$; Cohen's $d=-0.23$ ). They also showed 
Table 1 Correlations among the variables and descriptive statistics

\begin{tabular}{|c|c|c|c|c|c|c|c|c|}
\hline Variables & PI & NRM & PSM & CT & $\mathrm{CCC}$ & EQ-5D & $\begin{array}{l}\text { EQ- } \\
\text { VAS }\end{array}$ & $M \pm S D$ \\
\hline 1. Productive interactions $(\mathrm{PI})^{\mathrm{a}}$ & 1 & & & & & & & $4.44 \pm 0.71$ \\
\hline $\begin{array}{l}\text { 2. New relational model } \\
(\mathrm{NRM})^{\mathrm{a}}\end{array}$ & $0.29^{* *}$ & 1 & & & & & & $2.00 \pm 1.04$ \\
\hline $\begin{array}{l}\text { 3. Patient self-management } \\
(\text { PSM })^{\mathrm{a}}\end{array}$ & $0.73^{* *}$ & $0.40^{* *}$ & 1 & & & & & $4.12 \pm 0.79$ \\
\hline $\begin{array}{l}\text { 4. Complexity of treatment } \\
(\mathrm{CT})^{\mathrm{b}}\end{array}$ & $-0.16^{* *}$ & -0.04 & $-0.11^{*}$ & 1 & & & & $1.42 \pm 0.53$ \\
\hline $\begin{array}{l}\text { 5. Complexity of clinical care } \\
(\mathrm{CCC})^{c}\end{array}$ & $-0.21^{* *}$ & -0.05 & $-0.18^{* *}$ & $0.41^{* *}$ & 1 & & & $5.83 \pm 4.81$ \\
\hline $\begin{array}{l}\text { 6. Health-related quality of life } \\
(\text { EQ-5D })^{\mathrm{a}}\end{array}$ & $0.16^{* *}$ & 0.08 & $0.14^{* *}$ & $-0.39^{* *}$ & $-0.42^{* *}$ & 1 & & $4.51 \pm 0.59$ \\
\hline $\begin{array}{l}\text { 7. Self-rate of health (EQ- } \\
\text { VAS) }\end{array}$ & $0.26^{* *}$ & $0.14^{* *}$ & $0.28^{* *}$ & $-0.42^{* *}$ & $-0.36^{* *}$ & $-0.57^{* *}$ & & $73.25 \pm 19.13$ \\
\hline
\end{tabular}

Correlations were analyzed using Pearson's coefficients $(r)$

$M$ mean, $S D$ standard deviation, $P I$ productive interactions, NRM new relational model, PSM patient self-management, $E Q-5 D$ five dimensions of the Health-Related Quality of Life Questionnaire, EQ-VAS analog visual scale measuring self-rate of health, $C T$ complexity of the treatment, $C C C$ complexity of the clinical care

${ }^{*} p<0.01 ;{ }^{* *} p<0.001$

a The scales have a range of 5 points

b The variable complexity of treatment has a range of 4 points (it averages the number of diary doses ranged from 1 to 4 and the number of different medicines, which was rescaled to 4 points)

c The variable complexity of patient's clinical care averages the number of different physicians seeing the patient and number of different health-care sites that the patient usually attends

d The scale has a range of scores between 0 and 100 points

significantly higher scores in their treatment complexity $\quad(1.54 \pm 0.55 \quad$ vs. $1.24 \pm 0.38$; $t=-5.85 ; p<0.0001 ;$ Cohen's $d=0.61)$ and clinical care complexity $(6.60 \pm 5.09$ vs. $5.17 \pm 4.18 ; \quad t=-2.82 ; \quad p=0.005 ; \quad$ Cohen's $d=0.30$ ) than the younger group. Lastly, they also showed the lowest score in the overall perception of health $(69.74 \pm 19.05$ vs. 79.05 vs. $17.54 ; t=4.57 ; \quad p<0.0001 ; \quad$ Cohen's $d=-0.50)$.

\section{Model Testing}

Step 1: The influence of the experience of people living with HIV with healthcare on their health-related quality of life.
The first step was to test whether health-related quality of life could be predicted by the different dimensions of the patient experience (Fig. 1). Results showed that patient self-management was the only factor that was positively and significantly associated with both healthrelated quality of life measures (EQ-5D-5L: $\beta=0.34, p<0.0001$; and self-rated health, EQVAS: $\beta=0.38, p<0.0001)$. The relationship between the productive interactions and the new relational model with the two health-related quality of life measures was nonsignificant, although in the former (productive interactions with health-related quality of life), the path coefficients showed a negative, albeit minimal, direction (productive interactions EQ- 


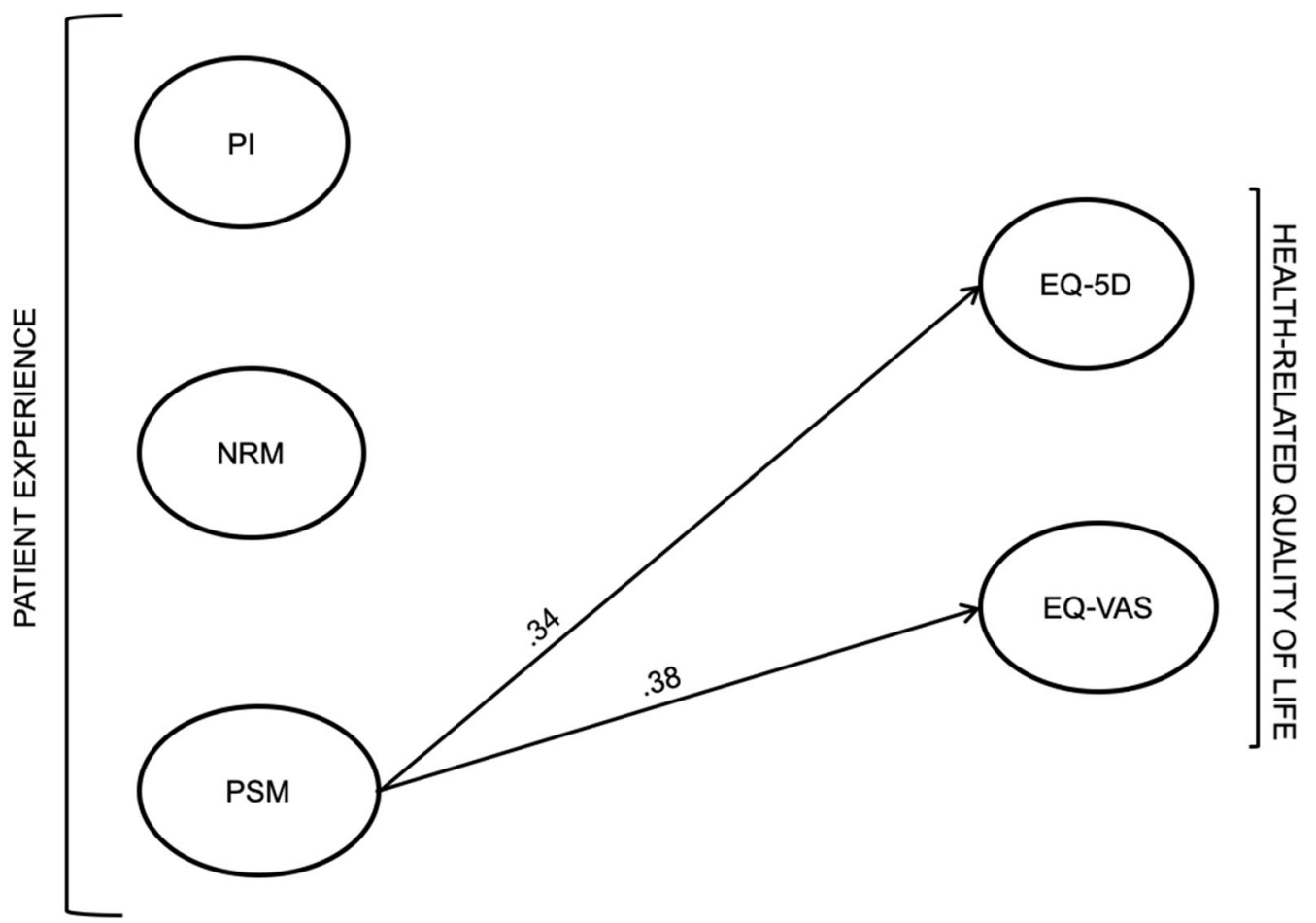

Fig. 1 Results of the partial least squares inner model showing the predictive relationships between the dimensions of patient experience and health-related quality of life (step 1 of the model testing). Standardized solution. For the sake of clarity, only the significant path coefficients are displayed in the figure. Student $t$ statistics and $p$ values of

5D-5L: $\beta=-0.07, p=0.26$; productive interactions self-rate of health, EQ-VAS: $\beta=-0.00$, $p=0.97$; new relational model EQ-5D-5L: $\beta=0.02, p=0.63$, and new relational model EQ-VAS: $\beta=0.05, \quad p=0.21)$. The model explained more variance in the self-rated health-EQ-VAS $\left(R^{2}=0.16\right)$ than that of the EQ-5D-5L $\left(R^{2}=0.09\right)$.

Summarizing the first step of the model testing, we can conclude that only the dimension of patient-self management positively and significantly predicted people living with HIVs' health-related quality of life.

Step 2: The influence of treatment complexity on health-related quality of life.

In the second step, we introduced treatment complexity into the model (Fig. 2). Results showed that it was negatively associated with both measures of health-related quality of life the path coefficients $(\beta)$ are displayed in Table 3. PI productive interactions, $N R M$ new relational model, $P S M$ patient self-management, $E Q-5 D$ five dimensions of the Health-related Quality of Life Questionnaire, EQ-VAS analog visual scale measuring self-rate of health

(EQ-5D-5L: $\beta=-0.37, p<0.0001$, and selfrated health (EQ-VAS): $\beta=-0.36, p<0.0001$ ). The introduction of treatment complexity in the model increased the amount of explained variance of health-related quality of life, showing a medium effect size in both the explanation of the 5D-5D-5L scale $\left(R^{2}=0.22 ; f^{2}=0.17\right)$ and self-rated health scale (EQ-VAS) $\left(R^{2}=0.29\right.$; $\left.f^{2}=0.18\right)$. Thus, this model had a higher predictive capacity of the people with HIV healthrelated quality of life than the previous one (model tested in Step 1).

Step 3: The influence of the complexity of clinical care on the model.

Finally, we introduced the complexity of clinical care into the model (Fig. 3). Results showed that it was negatively associated with the productive interactions and patient selfmanagement factors of the patient experience 


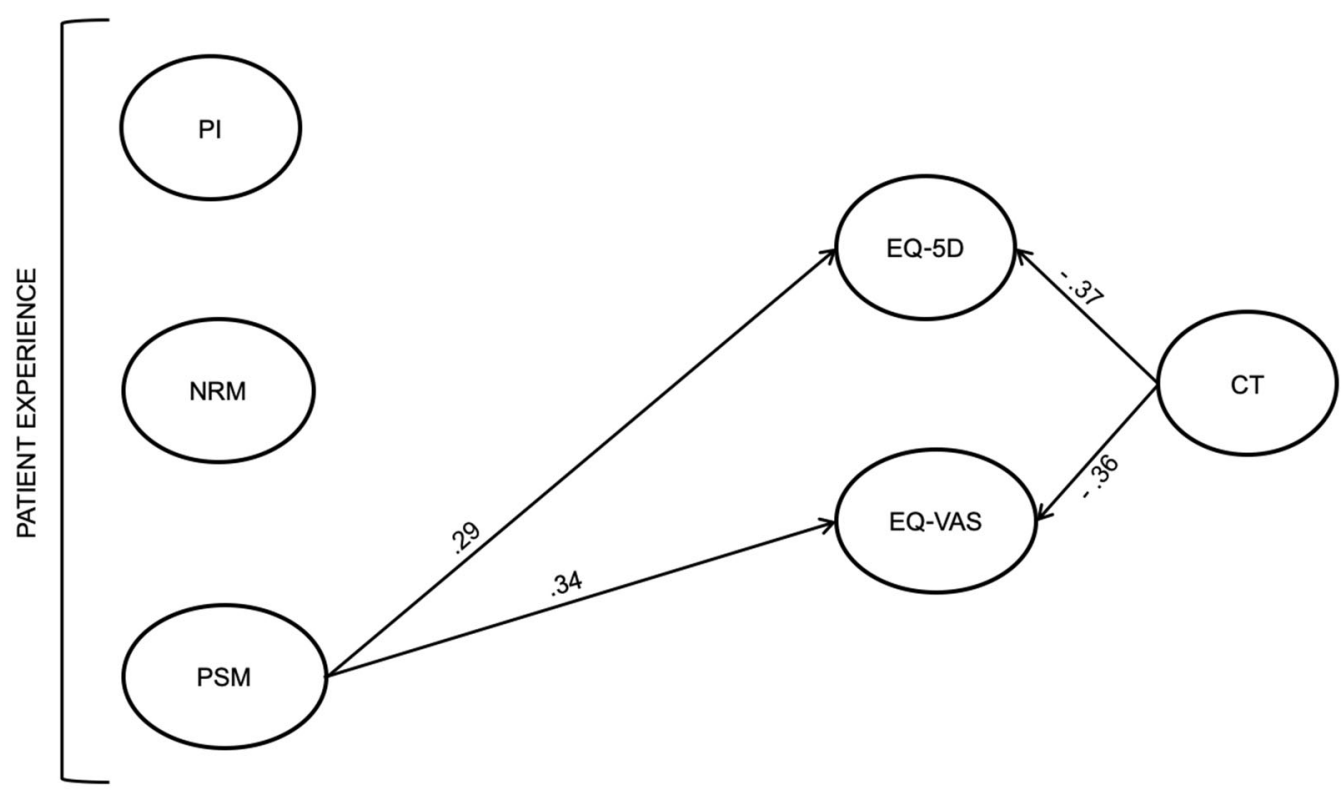

Fig. 2 Results of the partial least squares inner model showing the predictive relationship of the treatment complexity and health-related quality of life (step 2 of the model testing). Standardized solution. For the sake of clarity, only the significant path coefficients are displayed in the figure. Student $t$ statistics and $p$ values of the path

$(\beta=-0.21, \quad p<0.0001, \quad$ and $\quad \beta=-0.21$, $p<0.0001$, respectively). Complexity of clinical care was also negatively associated with both health-related quality of life measures (EQ-5D5L: $\beta=-0.37, p<0.0001$, and self-rated health (EQ-VAS): $\beta=-0.19, p<0.0001)$. Moreover, the negative relationship between the complexity of clinical care and the productive interactions factor led to an increased negative relationship of this factor on the EQ-5D-5L measure of health-related quality of life. The introduction of the complexity of clinical care in the model increased the amount of explained variance of the EQ-5D-5L health-related quality of life measure, showing a medium effect size $\left(R^{2}=0.32, f^{2}=0.17\right)$. However, the effect size was weak in the case of self-rated health (EQVAS $)\left(R^{2}=0.31, f^{2}=0.03\right)$. Thus, this model had a higher predictive capacity of the people with HIV's health-related quality of life than the two previous ones (models tested in step 1 and step 2). coefficients $(\beta)$ are displayed in Table 3. PI productive interactions, NRM new relational model, PSM patient selfmanagement, $E Q-5 D$ five dimensions of the Health-related Quality of Life Questionnaire, EQ-VAS analog visual scale measuring self-rate of health, $C T$ complexity of the treatment

Summarizing the results of the final model, we could observe that patient self-management showed the highest positive relationship with the patient's health-related quality of life. On the contrary, the productive interactions presented a negative relation with the EQ-5D-5L measure of health-related quality of life. Furthermore, the complexity of patients' treatment also predicted their health-related quality of life negatively. Finally, the complexity of clinical care also damaged healthrelated quality of life, both directly and through its negative influence on the productive interactions with healthcare professionals and patient self-management dimensions of the patient experience. The introduction in the model of the complexity of treatment and clinical care increased the predictive capacity of the model. Table 2 displays the total effects of the variables on the health-related quality of life.

For the sake of clarity, only the relationships among the latent variables (inner model) are 


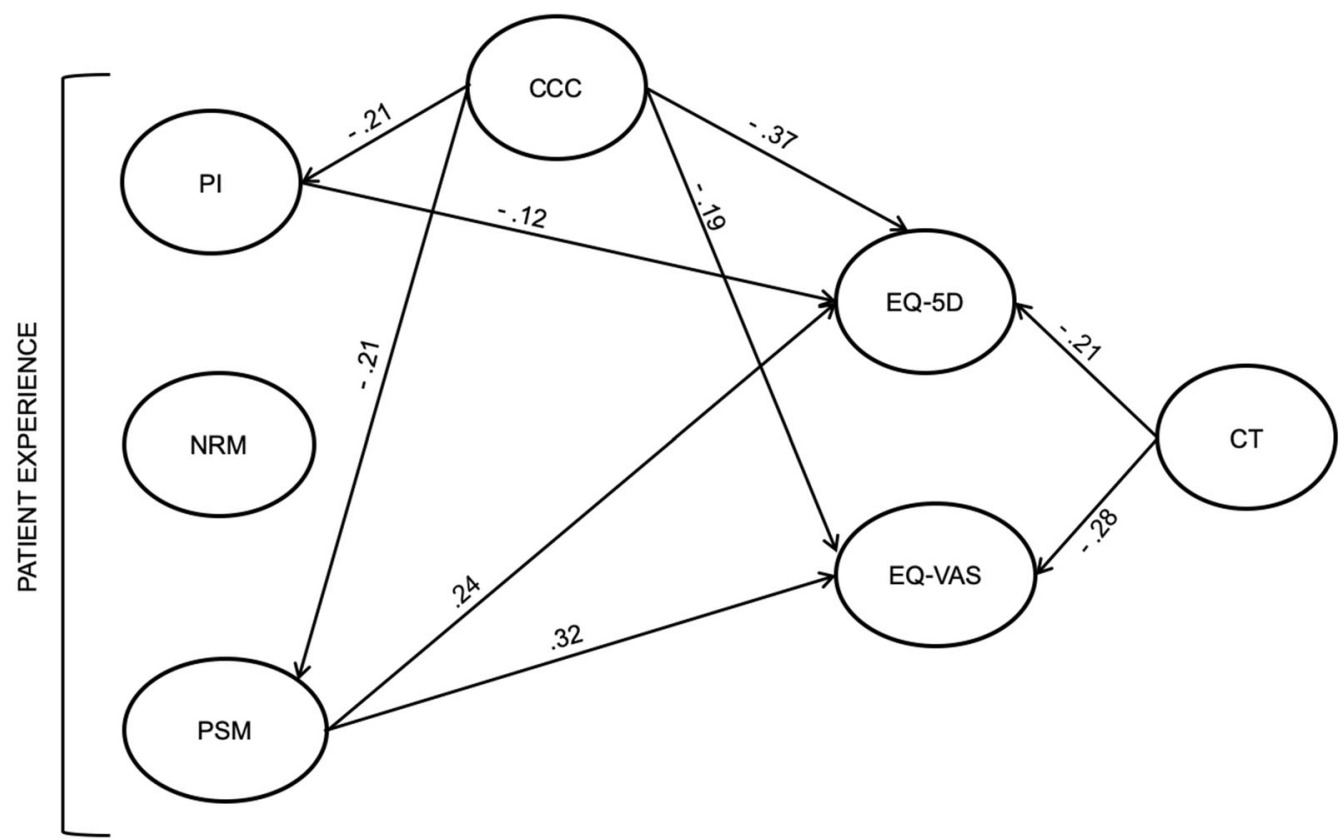

Fig. 3 Results of the final partial least squares inner model showing the predictive relationships among the studied variables after introducing the complexity of the clinical care into the model (step 3 of the model testing). Standardized solution. For the sake of clarity, only the significant path coefficients are displayed in the figure. Student $t$ statistics and $p$ values of $\beta$ coefficients are displayed in Table 3. PI productive interactions, NRM new relational model, PSM patient self-management, $E Q-5 D$ five dimensions of the Health-related Quality of Life Questionnaire, EQ-VAS analog visual scale measuring self-rate of health, $C T$ complexity of the treatment, $C C C$ complexity of the clinical care

Table 2 Total effects of the variables on participant's health-related quality of life considering the total sample and agespecific groups

\begin{tabular}{|c|c|c|c|c|c|c|}
\hline \multirow[t]{3}{*}{ Latent variables } & \multicolumn{6}{|c|}{ Total effects ${ }^{a}$} \\
\hline & \multicolumn{2}{|c|}{ Total sample } & \multicolumn{2}{|c|}{$\leq 49$ years } & \multicolumn{2}{|c|}{$\geq 50$ years } \\
\hline & $\overline{E Q-5 D}$ & VAS & $\overline{E Q-5 D}$ & VAS & $\overline{\text { EQ-5D }}$ & VAS \\
\hline Productive interactions & -0.12 & -0.04 & -0.17 & -0.06 & -0.16 & -0.17 \\
\hline New relational model & 0.03 & 0.05 & 0.03 & 0.06 & 0.11 & 0.11 \\
\hline Patient self-management & 0.24 & 0.32 & 0.17 & 0.18 & 0.28 & 0.46 \\
\hline Complexity of treatment & -0.28 & -0.21 & -0.32 & -0.28 & -0.16 & -0.23 \\
\hline Complexity of clinical care & -0.40 & -0.25 & -0.43 & -0.35 & -0.38 & -0.26 \\
\hline$R^{2}$ (explained variance) & 0.32 & 0.31 & 0.31 & 0.41 & 0.28 & 0.34 \\
\hline
\end{tabular}

$E Q-5 D$ five dimensions of the Health-related Quality of Life Questionnaire, EQ-VAS analog visual scale of Health-related Quality of Life Questionnaire measuring self-rate of health

a Total effects are the statistics displayed in the table showing the total effects (multiplication of direct and indirect effects) of the predictors on health-related quality of life 
Table 3 Results of the partial least square algorithm and bootstrapping of the final model in the total sample and in the agespecific studied groups

\begin{tabular}{|c|c|c|c|c|c|c|c|c|c|}
\hline \multirow[t]{2}{*}{ Path coefficients } & \multicolumn{3}{|c|}{ Total sample } & \multicolumn{3}{|c|}{$\leq 49$ years } & \multicolumn{3}{|c|}{$\geq 50$ years } \\
\hline & $\bar{\beta}$ & $t$ & $p$ & $\bar{\beta}$ & $t$ & $p$ & $\bar{\beta}$ & $t$ & $p$ \\
\hline Productive interactions EQ-5D & -0.12 & 2.06 & 0.039 & -0.17 & 1.45 & 0.146 & -0.16 & 1.96 & 0.050 \\
\hline Productive interactions EQ-VAS & -0.04 & 0.81 & 0.416 & -0.06 & 0.54 & 0.588 & -0.17 & 2.11 & 0.035 \\
\hline New relational model EQ-5D & 0.03 & 0.88 & 0.377 & 0.04 & 0.45 & 0.653 & 0.11 & 2.08 & 0.037 \\
\hline New relational model EQ-VAS & 0.05 & 1.25 & 0.210 & 0.06 & 0.64 & 0.519 & 0.11 & 1.93 & 0.054 \\
\hline Patient self-management EQ-5D & 0.24 & 4.16 & $<0.0001$ & 0.17 & 1.29 & 0.195 & 0.28 & 3.41 & 0.001 \\
\hline Patient self-management EQ-VAS & 0.32 & 5.57 & $<0.0001$ & 0.18 & 1.42 & 0.156 & 0.46 & 5.49 & $<0.0001$ \\
\hline Complexity of treatment EQ-5D & -0.21 & 4.12 & $<0.0001$ & -0.32 & 3.74 & $<0.0001$ & -0.15 & 2.47 & 0.014 \\
\hline Complexity of treatment EQ-VAS & -0.28 & 6.50 & $<0.0001$ & -0.28 & 4.05 & $<0.0001$ & -0.23 & 3.21 & 0.001 \\
\hline Complexity of clinical care EQ-5D & -0.37 & 7.26 & $<0.0001$ & -0.41 & 5.02 & $<0.0001$ & -0.35 & 4.91 & $<0.0001$ \\
\hline Complexity of clinical care EQ-VAS & -0.19 & 4.41 & $<0.0001$ & -0.31 & 3.70 & $<0.0001$ & -0.20 & 3.02 & 0.003 \\
\hline $\begin{array}{l}\text { Complexity of clinical care productive } \\
\text { interactions }\end{array}$ & -0.21 & 4.58 & $<0.0001$ & -0.20 & 2.27 & 0.024 & -0.18 & 3.03 & 0.002 \\
\hline $\begin{array}{l}\text { Complexity of clinical care patient self- } \\
\text { management }\end{array}$ & -0.21 & 4.87 & $<0.0001$ & -0.31 & 3.61 & $<0.0001$ & -0.19 & 2.82 & 0.005 \\
\hline
\end{tabular}

$\beta$ standardized beta coefficients, $t$ Student's $t, E Q-5 D$ five dimensions of Health-related Quality of Life Questionnaire, $E Q$ $V A S$ analog visual scale of Health-related Quality of Life Questionnaire measuring self-rate of health

displayed in the figures. Table 3 shows all the statistics and parameters of the partial least square algorithm and bootstrapping of the structural model. The results of the relationships among the latent variables, and its item indicators (measurement model) are provided as Electronic Supplementary Material 1. Results showed that the loadings of most indicators of the latent variables $(\lambda)$ were $>0.50$, which can be considered adequate [17]. Most variables also presented adequate construct reliability and validity. The factor "new relational model" had the lowest reliability, although it could be considered adequate, as it had only three indicators [22]. The patient self-management factor showed the lowest value of convergent validity, but it was close to 0.50 [average variance extracted $(\mathrm{AVE})=0.44]$, a value that can be considered adequate [17].

\section{Differences According to Age in the Predictive Model}

To test whether the model was invariant or equivalent across the established age-specific groups ( $\mathrm{G} 1 \leq 49$ years, $\mathrm{G} 2 \geq 50$ years), we first assessed the measured model invariance. Configurational invariance was assumed because constructs were equally parameterized and estimated across groups. Regarding compositional invariance, results of the measurement invariance of the composite model procedure showed that the correlations between the composite scores of the two groups were not significantly $<1$ in most variables except for treatment complexity (permutation $p=0.022$ ). Thus, partial measurement invariance or equivalence cannot be established for this latent variable. Results of the assessment of 
compositional invariance procedure are provided in Electronic Supplementary Material 2.

Consequently, we analyzed the predictive model separately in the two age-specific subsamples. Results showed that the path coefficients among the three factors of the patient experience and both measures of the healthrelated quality of life (EQ-5D-5L and EQ-VAS) were only significant in those people living with HIV $\geq 50$ years (Table 3 ). Thus, the total effects of these factors of patient experience on healthrelated quality of life were higher in the people living with $\mathrm{HIV} \geq 50$ years than in those $\leq 49$ years (Table 2 ), and they were significant only in the older group $(p<0.05)$. The highest effect size in this group was the positive relationship between the factor patient selfmanagement and self-rated health $\left(f^{2}=0.16\right)$. However, the complexity of treatment and clinical care produced the highest total effects in the people living with HIV $\leq 49$ years, and the path coefficients between these variables and the health-related quality of life EQ-5D-5L measure had the highest effect sizes in this group $\left(f^{2}=0.15\right.$ and $f^{2}=0.23$ for the complexity of treatment and the complexity of clinical care, respectively).

\section{DISCUSSION}

The present study aimed to analyze the influence of different factors of patients' experience on the health-related quality of life of people living with HIV and the role that the complexity of the treatment and clinical care played in it. Furthermore, it analyzed differences in those relationships according to whether the participants were younger or older than 50 years, which is a suggested cutoff point to consider the health impact of aging in people living with HIV [4].

Results of the model tested in the total sample showed that patient self-management was the only dimension of patients' experience that positively predicted the health-related quality of life of people living with HIV. Several studies have shown the central role that patient self-management plays in the health and wellbeing of patients living with chronic diseases
$[23,24]$. Programs of training patients in selfmanagement behaviors led to an improvement of health status, reduction of hospital admissions, and the costs of healthcare [23]. Such training also improves healthy behaviors and health outcomes of people living with HIV $[25,26]$.

However, patients' experience itself explained a limited amount of the variance of the health-related quality of life. It is because treatment and clinical care complexity played a relevant role in their relationship. The treatment complexity of people living with HIV had a direct negative association with their healthrelated quality of life. Previous studies have found a negative influence of complex treatments in people living with HIV wellbeing $[27,28]$ and the risks of polypharmacy in aging people living with HIV who are suffering comorbidities [4]. The complexity of patients with HIV's clinical care also had a direct negative influence on their health-related quality of life. However, it also damaged their health-related quality of life indirectly through its negative effects on the productive interactions of people living with HIV with healthcare professionals and their health self-management. The complexity of clinical care might make interactions with healthcare providers negative or unproductive and hinder the self-management of their health. Previous results from our broader research showed that some variables related to the complexity of clinical care were negatively associated with all factors of the different chronic patients' experience [13]. Our secondary analysis showed their impact not only on HIV patients' experience but also on their health-related quality of life.

Our results also showed that the effects of the dimensions of patient experience on their health-related quality of life were higher in people living with HIV older than 50 years than those who were younger. The highest positive influence of patient self-management on health-related quality of life occurred in this group. Moreover, the new relational model influenced the health-related quality of life in those older than 50 years but not of the younger patients. The management of the HIV aging process implies an increased challenge for both 
patients and healthcare providers [29, 30]. According to our results, it seems that patients' experience with healthcare professionals is particularly relevant in the management of the aging process of people living with HIV. There is currently a scientific debate on the need for a new healthcare model that responds effectively to the emerging challenges related to the aging process of people living with HIV $[4,31]$. Lessons learned from the management of other chronic diseases point out the need to move toward patient-centered models such as the Chronic Care Model [24].

Results also showed that, although people living with HIV older than 50 years presented higher scores in the complexity of treatment and clinical care than those younger than 50, such complexity produced a higher negative effect on the health-related quality of life of the latter group ( $\leq 49$ years). A possible explanation for this increased burden in younger patients' health-related quality of life may be that they have less mastery and skills to deal with such complexity and they are less resilient than the older patients, who had probably lived more time with HIV and other comorbidities. Mastery, acceptance of illness, self-care, and illness perception were some of the factors found to be related to resilience in the physically ill, and some studies found that old age was related to being more adaptive and resilient [32].

Our study has some limitations: first, those derived from its cross-sectional nature, which precludes establishing causal relationships among the variables under study. Future studies should confirm our results using a longitudinal approach. Also, we did not perform probabilistic sampling, but the consecutive inclusion of patients aimed to reduce selection bias. Moreover, because the survey was anonymous, we could not know the profiles of the people living with HIV who did not return the survey. A larger sample is necessary to strengthen the comparisons according to age and generalize the results. Besides, time since HIV diagnosis was not collected because it was neither the primary nor secondary objective of the study, and the research team decided to collect the minimum data to facilitate quick answers of the participants and thus get a large sample. However, time since HIV diagnosis could have an impact on the patient's experience and their quality of life. Some of the results found in our study related to age could reflect not only the effect of age, but also their interaction with time since HIV diagnosis. Future studies should deeply analyze the effects of time living with HIV in the patient experience and their quality of life.

\section{CONCLUSION}

In summary, the present study found that patients' experience with healthcare was associated mainly with the health-related quality of life of aging of people living with VIH. Patient self-management showed the highest positive relation with their health-related quality of life. Treatment and clinical care complexity played an important role in damaging patients' experience and their health-related quality of life. The World Health Organization recognizes patient self-management as the best practice to improve clinical care and outcomes in chronic patients [33]. Our results highlight the need for more integrated care for people living with HIV because it has been shown to have beneficial effects on several chronic health-related outcomes and on the quality of patient care [34].

\section{ACKNOWLEDGEMENTS}

The authors wish to thank all patients who completed the survey and the IEXPAC working group for providing the valuable tool. This study was partially presented at HIV Glasgow 2018 (Glasgow, UK; 28-31 October 2018) and at the $\mathrm{X}$ Congreso Nacional GESIDA (Madrid, Spain; 6-9 November 2018).

Funding. This study was funded by Merck Sharp \& Dohme of Spain, a subsidiary of Merck \& Co., Inc., Kenilworth, NJ, USA. The study was reviewed and endorsed by the following patients' associations: Spanish Association of Patients with Arthritis (CONARTRITIS); Spanish Association of Patients with Crohn's Disease and Ulcerative Colitis (ACCU); the Spanish 
Federation of Patients with Diabetes (FEDE); the Spanish AIDS Multidisciplinary Society (SEISIDA). Merck Sharp \& Dohme of Spain provided funding to SEISIDA for medical writing and editorial assistance and for covering the expenses of the manuscript publication.

Authorship. All named authors meet the International Committee of Medical Journal Editors (ICMJE) criteria for authorship for this article, take responsibility for the integrity of the work as a whole, and have given their approval for this version to be published.

Authorship Contributions. MJF-R assumed primary responsibility for analyzing data and writing the manuscript. NS-V and MC participated in the design and implementation of the study. The rest of the authors made substantial contributions to the manuscript.

Disclosures. María J. Fuster-RuizdeApodaca has provided consultancy services to ViiV, Gilead, and Janssen; her institution has received grants from MSD, ViiV, Janssen, and Gilead and payments for lectures or educational presentations from MSD, Gilead, and ViiV. Nuria Sánchez-Vega is a full-time employee at Merck Sharp \& Dohme de España; SA and may own stock and/or stock options. Manuel Cotarelo is a full-time employee at Merck Sharp \& Dohme de España, SA, and may own stock and/or stock options. María J. Galindo has provided consultancy services to ViiV, Abbie, MSD, Gilead, and Janssen; her institution has received grants from MSD, Janssen, and Gilead and payments for traveling to meetings from MSD and for lectures or educational presentations from MSD, Janssen, Gilead, and ViiV. Ignacio Marin-Jimenez has served as a consultant, member of advisory boards, received travel grants or economic support for research from Abbvie, MSD, Takeda, Janssen, Dr. Falk Pharma, Ferring, Shire, Sandoz, Fresenius, and Pfizer. Juan Carlos López has received payments for traveling to meetings from MSD, ViiV, and Gilead and for lectures or educational presentations from MSD, Janssen, Gilead, and ViiV. Javier de Toro and Domingo Orozco-Beltrán have nothing to declare.
Compliance with Ethics Guidelines. The Clinical Investigation Ethics Committee of the Gregorio Marañón Hospital of Madrid (Spain) approved the research protocol. The study documentation included printed instructions and information for patients about the anonymous nature of the survey and aggregated data processing, which ensured that patient identification was not possible. As agreed by the Clinical Investigation Ethics Committee, the voluntary return of completed questionnaires was taken as implied consent to participate in the study. No clinical data were collected in this study.

Data Availability. The datasets generated during and/or analyzed during the current study are available from the corresponding author on request. All authors had full access to all of the data in this study and take complete responsibility for the integrity of the data and accuracy of the data analysis.

Open Access. This article is distributed under the terms of the Creative Commons Attribution-NonCommercial 4.0 International License (http://creativecommons.org/licenses/ by-nc/4.0/), which permits any noncommercial use, distribution, and reproduction in any medium, provided you give appropriate credit to the original author(s) and the source, provide a link to the Creative Commons license, and indicate if changes were made.

\section{REFERENCES}

1. Joint United Nations Programme on HIV/AIDS (UNAIDS). UNAIDS DATA 2017. Geneva. 2017. http://www.unaids.org/sites/default/files/media_ asset/20170720_Data_book_2017_en.pdf. Accessed July 1, 2018.

2. Area de Vigilancia del VIH y comportamientos de riesgo. Vigilancia epidemiológica del VIH y sida en España 2016: sistema de información sobre nuevos diagnósticos de VIH y Registro nacional de casos de sida. Madrid. 2017. https://www.msssi.gob.es/ ciudadanos/enfLesiones/enfTransmisibles/sida/ vigilancia/InformeVIH_SIDA_2017_NOV2017.pdf. Accessed July 1, 2018. 
3. del Amo J, Pérez-Molina JA. Introducción. La infección por VIH en España: situación actual y propuestas frente a los nuevos desafíos. Enferm Infecc Microbiol Clin. 2018;36(Supl 1):50-1.

4. Berenguer J, Álvarez D, Dodero J, Azcoaga A. Modelo de seguimiento, organización y gestión de la infección por VIH. Enferm Infecc Microbiol Clin. 2018;36(Supl 1):45-9.

5. Centro Nacional de Epidemiología, Instituto de Salud Carlos III, Plan Nacional sobre el Sida-SG de Promoción de la Salud y Epidemiología. Encuesta Hospitalaria de pacientes con infección por el VIH: Resultados 2016. Análisis de la evolución 2001-2016. Madrid. 2017. https://www.mscbs.gob. es/ciudadanos/enfLesiones/enfTransmisibles/sida/ vigilancia/InformeEncuestaHospitalaria2016DEF. pdf. Accessed July 10, 2018.

6. Miners A, Phillips A, Kreif N, Rodger A, Speakman A, Fisher M, et al. Health-related quality-of-life of people with HIV in the era of combination antiretroviral treatment: a cross-sectional comparison with the general population. Lancet HIV. 2014;1(1):e32-40.

7. Emuren L, Welles S, Polansky M, Evans AA, Macalino G, Agan BK, et al. Lower health-related quality of life predicts all-cause hospitalization among HIVinfected individuals. Health Qual Life Outcomes. 2018;16(1):107.

8. Lazarus JV, Safreed-Harmon K, Barton SE, Costagliola D, Dedes N, del Amo Valero J, et al. Beyond viral suppression of HIV-the new quality of life frontier. BMC Med. 2016;14(1):94.

9. Greene ML, Tan JY, Weiser SD, Christopoulos K, Shiels M, O'Hollaren A, et al. Patient and provider perceptions of a comprehensive care program for HIV-positive adults over 50 years of age: the formation of the Golden Compass HIV and aging care program in San Francisco. PLoS One. 2018;13(12):e0208486.

10. Mira JJ, Nuño-Solinís R, Guilabert-Mora M, SolasGaspar O, Fernández-Cano P, González-Mestre MA, et al. Development and validation of an instrument for assessing patient experience of chronic illness care. Int J Integr Care. 2016;16(3):1-13.

11. Cramm JM, Nieboer AP. High-quality chronic care delivery improves experiences of chronically ill patients receiving care. Int $\mathrm{J}$ Qual Heal Care. 2013;25(6):689-95.

12. Doyle C, Lennox L, Bell D. A systematic review of evidence on the links between patient experience and clinical safety and effectiveness. BMJ Open. 2013;3(1):e001570.
13. Orozco-Beltrán D, de Toro J, Galindo MJ, MarínJiménez I, Casellas F, Fuster-RuizdeApodaca MJ, et al. Healthcare experience and their relationship with demographic, disease and healthcare-related variables: a cross-sectional survey of patients with chronic diseases using the IEXPAC scale. Patient Patient Centered Outcomes Res. 2018. https://doi. org/10.1007/s40271-018-0345-1.

14. Devlin NJ, Brooks R. EQ-5D and the EuroQol group: past, present and future. Appl Health Econ Health Policy. 2017;15(2):127-37.

15. Herdman M, Gudex C, Lloyd A, Janssen M, Kind P, Parkin D, et al. Development and preliminary testing of the new five-level version of EQ-5D (EQ-5D5L). Qual Life Res. 2011;20(10):1727-36.

16. Hair JF, Ringle CM, Sarstedt M. Partial least squares structural equation modeling: rigorous applications, better results and higher acceptance. Long Range Plann. 2013;46(1-2):1-12.

17. Henseler J, Ringle CM, Sinkovics RR. The use of partial least squares path modeling in international marketing. In: Rudolf R, Sinkovics PNG, editors. New challenges to international marketing. Bingley: Emerald Group Publishing Limited; 2009. p. 277-319.

18. Henseler J, Chin WW. A comparison of approaches for the analysis of interaction effects between latent variables using partial least squares path modeling. Struct Equ Model A Multidiscip J. 2010;17(1): 82-109.

19. Falk R, Miller NB. A primer for soft modeling. Akron: University of Akron Press; 1992.

20. Chin WW. The partial least squares approach for structural equation modeling. In: Marcoulides GA, editor. Modern methods for business research. New York: Psychology Press; 1998. p. 295-336.

21. Henseler J, Ringle CM, Sarstedt M. Testing measurement invariance of composites using partial least squares. Int Mark Rev. 2016;33(3):405-31.

22. Nunnally J, Bernstein I. Psychometric theory. 3rd ed. New York: McGraw-Hill; 1994.

23. Lorig KR, Holman H. Self-management education: history, definition, outcomes, and mechanisms. Ann Behav Med. 2003;26(1):1-7.

24. Coleman K, Austin BT, Brach C, Wagner EH. Evidence on the chronic care model in the new millennium. Health Aff. 2009;28(1):75-85.

25. Marshall R, Beach MC, Saha S, Mori T, Loveless MO, Hibbard JH, et al. Patient activation and improved 
outcomes in HIV-infected patients. J Gen Intern Med. 2013;28(5):668-74.

26. Millard T, Elliott J, Girdler S. Self-management education programs for people living with HIV/ AIDS: a systematic review. AIDS Patient Care STDS. 2013;27(2):103-13.

27. Park-Wyllie LY, Strike CS, Antoniou T, Bayoumi AM. Adverse quality of life consequences of antiretroviral medications. AIDS Care. $2007 ; 19(2): 252-7$.

28. Ventura Cerdá JM, Casado Gómez MA, Escobar Rodríguez I, Ibarra Barrueta O, Ortega Valín L, Morales González JM, et al. Preferencias, satisfacción y adherencia con el tratamiento antirretroviral: estudio ARPAS (II). Farm Hosp. 2007;31(6):340-52.

29. Cardoso SW, Torres TS, Santini-Oliveira M, Marins LMS, Veloso VG, Grinsztejn B. Aging with HIV: a practical review. Braz J Infect Dis. 2013;17(4):464-79.

30. UNAIDS. HIV and aging: a special supplement to the UNAIDS report on the global AIDS epidemic
2013. Geneva. 2013. http://www.unaids.org/en/ media/unaids/contentassets/documents/ unaidspublication/2013/20131101_JC2563_hivand-aging_en.pdf. Accessed Sept 10, 2018.

31. Brañas F, Azcoaga A, García Ontiveros M, Antela A. Cronicidad, envejecimiento y multimorbilidad. Enferm Infecc Microbiol Clin. 2018;36(Supl $1): 15-8$.

32. Stewart DE, Yuen T. A systematic review of resilience in the physically ill. Psychosomatics. 2011;52(3):199-209.

33. Noncommunicable Diseases and Mental Health World Health Organisation. Innovative care for chronic conditions. Geneva: World Health; 2002. https://www.who.int/chp/knowledge/publications/ icccglobalreport.pdf. Accessed Sept 11, 2018.

34. Martínez-González NA, Berchtold P, Ullman K, Busato A, Egger M. Integrated care programmes for adults with chronic conditions: a meta-review. Int J Qual Health Care. 2014;26(5):561-70. 